\title{
Complete resection of a cavoatrial metastatic dedifferentiated liposarcoma under hypothermic circulatory arrest
}

\author{
Ryumon Matsumoto ${ }^{1}$, Koki Maekawa ${ }^{1}$, Toshiki Fujiyoshi ${ }^{2}$, Kentaro Kamiya ${ }^{3}$, Jun \\ Matsubayashi $^{1}$, Shoji Fukuda ${ }^{1}$, Toshiya Nishibe ${ }^{4}$, and Hitoshi Ogino ${ }^{3}$ \\ ${ }^{1}$ Affiliation not available \\ ${ }^{2}$ Pediatric Cardiovascular Surgery \\ ${ }^{3}$ Tokyo Medical University \\ ${ }^{4}$ Tokyo Medical University Hospital
}

April 20, 2021

\begin{abstract}
A patient had undergone surgical resection twice for primary and metastatic dedifferentiated liposarcomas. Follow-up computed tomography revealed a new tumor mass located at the cavoatrial junction. Prompt surgical resection of the tumor with thrombectomy was successfully performed using cardiopulmonary bypass with hypothermic circulatory arrest. We report a rare case of a patient surviving for 8 years after the first tumor resection despite the poor prognosis of metastatic or recurrent liposarcoma.
\end{abstract}

\section{Introduction}

Although liposarcoma is a common soft tissue sarcoma, pleural liposarcoma has rarely been reported and its prognosis is poor due to higher incidences of metastasis and recurrence ${ }^{1}$. We report the case of a patient who underwent successful complete resection of a cavoatrial metastatic tumor originating from primary pleural liposarcoma using cardiopulmonary bypass $(\mathrm{CPB})$ and hypothermic circulatory arrest (HCA). The patient is surviving without any metastasis or recurrence.

A 68-year-old man was referred to our department for resection of a tumor located at the cavoatrial junction between the inferior vena cava (IVC) and the right atrium (RA). At the age of 62 , he was diagnosed with lung cancer and underwent a left lower lobectomy. However, the final diagnosis of the resected tumor was pleural dedifferentiated liposarcoma as the pathological analysis was positive for murine double minute 2 (MDM2), adipophilin, and perilipin. In one year, surgical adrenalectomy was performed for the right adrenal tumor and was repeated one year later for the left-side tumor. The pathological examination of the bilateral adrenal tumors also showed dedifferentiated liposarcoma metastasizing from the primary pleural liposarcoma. Follow-up computed tomography scans after surgery revealed a new mass in the IVC. Direct oral anticoagulant was administered with the diagnosis of venous thromboembolism. Repeated computed tomography revealed enlargement of the mass, which was suspected to be a metastatic liposarcoma (Fig. 1 ) .

The patient was asymptomatic. Blood examinations revealed no significant abnormality. All tumor markers were negative. Transthoracic echocardiography revealed a $60-\mathrm{mm}$, very mobile, right atrial mass extending into the right ventricle through the tricuspid valve (Fig. 2 ).

Materials and Methods 
Resection of the cavoatrial tumor with thrombectomy was performed. CPB was established with ascending aortic cannulation and venous drainage from the right femoral vein with a 21-Fr long cannula and the superior vena cava. The tip of the femoral vein cannula was carefully adjusted in the proximal part of the IVC with a transesophageal echo guide to avoid contact with the tumor or thrombus. After CPB was established successfully, cardiac arrest with ascending aortic cross-clamping was induced by antegrade cardioplegia. The superior vena cava was snugged, and a right atriotomy was made. A yellowish granular tumor mass was observed in the RA extending distally into the IVC beyond the hepatic vein junction. It was difficult to remove the tumor completely in the bloody surgical field. Hence, with the IVC gently snugged, core cooling was initiated along with simultaneous surface head cooling with ice bags until targeted tympanic temperature of $25^{\circ} \mathrm{C}$ was achieved. HCA was then induced and the tumor was removed smoothly. Endoscopy was used to ensure complete resection of the tumor. The duration of HCA was 3 min. The patient was smoothly weaned from CPB using a low dose of catecholamine. The time of aortic cross-clamp, CPB, and surgery was 84,172 , and $304 \mathrm{~min}$, respectively.

Results

The resected tumor comprised pleomorphic tumor cells containing lipoblastic cells with vacuolated cytoplasms. Immunohistochemical analysis showed diffuse positivity for MDM2 in tumor cells. Histopathological diagnosis of the tumors was dedifferentiated liposarcoma (Fig. 3). On postoperative day 14, the patient was discharged and followed up without adjuvant chemotherapy or radiotherapy. He has survived for 8 years since the initial surgery and 2 years since the second surgery.

\section{Conclusions}

Liposarcomas commonly occur in the extremities, however, primary pleural liposarcoma is extremely rare with very few reported cases ${ }^{1}$. We could only find 34 reported cases of pleural liposarcomas ${ }^{1-4}$.

The World Health Organization classifies liposarcoma into five types: atypical, well-differentiated, dedifferentiated, myxoid, and pleomorphic ${ }^{1}$. Of these, the dedifferentiated liposarcomas have a significantly poor prognosis with rates of local recurrence and metastasis of $41 \%$ and $17 \%$, respectively, and 5-year survival rate of only $28 \%$. Conversely, the 5 -year survival rate for well-differentiated liposarcomas is $80 \%^{5}$. The curative treatment for liposarcoma is surgical resection, with chemotherapy and/or radiotherapy. Adjuvant chemotherapy is selectively applied because the subtypes differ in chemosensitivity; it is not indicated in the resistant subtypes of dedifferentiated liposarcomas. Radiation therapy reportedly reduces the risk of local recurrence, especially in cases with myxoid liposarcoma ${ }^{3}$.

Regarding surgical resection of the tumor at the cavoatrial junction, Neves and Zincke classified venous tumor thrombus into four categories: level I-renal vein thrombus, level II- infrahepatic IVC thrombus, level III-retrohepatic IVC thrombus, and level IV-intraatrial IVC thrombus. In the presented case, the cavoatrial tumor thrombus can be classified as level IV. The standard surgical technique for level IV tumor thrombus is resection with $\mathrm{CPB}$. However, the use of $\mathrm{CPB}$ in patients suffering from malignant diseases is controversial because $\mathrm{CPB}$ decreases the immunity of patients and potentially causes the spread of malignant cells to the whole body. Furthermore, CPB is associated with the release of inflammatory mediators, coagulopathy, platelet dysfunction, and increased bleeding. There have been contradictory reports on the use of CPB in cancer patients ${ }^{6,7}$. Among 74 patients with metastatic cancer undergoing open-heart surgery with and without $\mathrm{CPB}$, no significant difference was reportedly observed in cancer-specific mortality (patients with and without CPB: $26.7 \%$ and $24.1 \%$, respectively, $\mathrm{p}=0.8)^{6}$. Conversely, another report demonstrated among 43,347 patients undergoing isolated CABG surgery, the adjusted relative risk of cancer-specific mortality caused by CPB was 1.16 (95\% CI: $0.92-1.46, \mathrm{p}=0.20)^{7}$. The lack of significant association between CPB and the progression of malignant diseases allowed us to perform aggressive surgical resection with CPB.

Similarly, there are no reports on HCA in cases with cavoatrial tumors, particularly liposarcoma. It was reported that $\mathrm{CPB}$ with $\mathrm{HCA}$ to treat level III or IV renal or adrenal tumors enabling complete tumor resection did not increase operative risks and was relatively safe ${ }^{8}$. The HCA proved useful in removing the cavoatrial tumor completely and in viewing the inside of the IVC clearly with endoscopy. 
In conclusion, prompt complete resection of the cavoatrial metastatic dedifferentiated liposarcoma associated with poor prognosis was successfully performed using CPB with HCA, which resulted in satisfactory midterm survival without recurrence.

Acknowledgements: I acknowledge Jun Matsubayashi, a professor at Tokyo Medical University, Department of Pathology, for assisting with the pathological analysis of this case.

\section{References}

1. Matsukuma S, Oshika Y, Utsumi Y,ObaraK, TanimotoT, KatsuradaY, TakeoH. Pleural dedifferentiated liposarcoma: A case report. Mol Clin Oncol 2019;10:132-136.

2. Lin CJ, Chou SH, Yang SF, Kao CN, Chang PC, Liu YW. Rapidly growing pleural liposarcoma masquerading as extrapleural hematoma. Thorac Cancer 2019;10:365-368.

3. Prabhakar N, Vaiphei K, Vishwajeet V, Ramamoorthy E, Gorsi U, Dhooria S, Kapoor R, Sandhu MS. Primary pleural liposarcoma: A rare entity. Lung India 2019;36:438-440.

4. Kang LH, Hwang CS, Yoon SH. Primary pleural liposarcoma combined spindle cell lipoma of the lung. Thorac Cancer 2020;11:2059-2062.

5. Henricks WH, Chu YC, Goldblum JR, Weiss SW. Dedifferentiated LPS: A clinicopathological analysis of 155 cases with a proposal for an expanded definition of dedifferentiation. Am J Surg Pathol 1997;21:271-281.

6. Suzuki S, Usui A, Yoshida K, Matsuura A, Ichihara T, Ueda Y. Effects of cardiopulmonary bypass on cancer prognosis. Asian Cardiovasc Thorac Am 2010;18:536-540.

7. Pinto CA, Marcella S, August DA, Holland B, Kostis JB, Demissie K. Cardiopulmonary bypass has a modest association with cancer progression: A retrospective cohort study. BMC Cancer 2013;13:519.

8. Zhu P, Du S, Chen S,ZhengS, HuY, LiuL, ZhengS. The role of deep hypothermic circulatory arrest in surgery for renal or adrenal tumor with vena cava thrombus: A single-institution experience. J Cardiothorac Surg 2018;13:85.

\section{Figure legends}

Fig.1

Preoperative contrast-enhanced computed tomography scans showing a tumor located at the cavoatrial junction

Fig.2

Preoperative echocardiogram showing a large tumor in the right atrium prolapsing through the tricuspid valve into the right ventricle

RA-Right Atrium

RV-Right Ventricle

Fig.3

Macroscopic and microscopic appearance of the extirpated tumor

a: Macroscopic appearance of the extirpated tumor. b: Microscopic examination of the lesion demonstrated tumor cells containing lipoblastic cells with vacuolated cytoplasms characteristic to liposarcoma (HE: High power field).

c: Immunohistochemically, the tumor cells showing diffuse positivity for MDM2 (HE: High power field) 

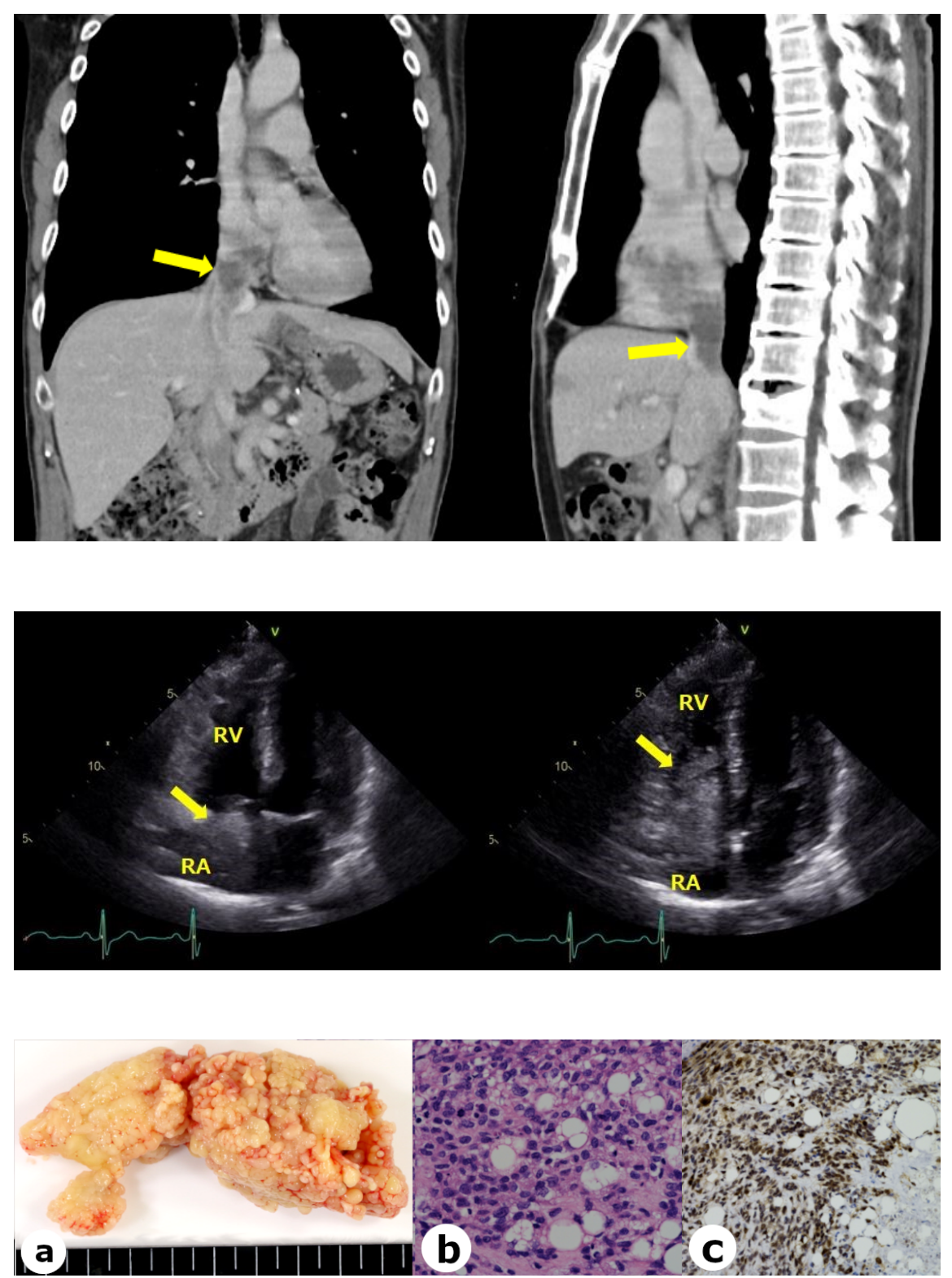